\title{
New algorithm for autonomous dynamic path planning in real-time intelligent robot car
}

\begin{abstract}
Finding an optimal path with minimum cost and process execution time is a major problem in dynamic environment where static and moving obstacles exist. Different algorithms have been used to address this problem by considering the optimal path with minimum cost; however, these algorithms did not consider the execution time to find such path. An intelligent design method for multipath planning in autonomous robot car is proposed in this work. Through the use of modified $\mathrm{A}^{*}$ algorithm, the optimal path with a minimum cost can be determined and an efficient execution time of moving from a starting location to a target location in a dynamic environment can be achieved. Distance, process execution time, and optimal path factors are considered to determine the cost of path planning. The proposed algorithm was simulated and tested using MATLAB with Virtual Robot Experimentation Platform. The simulation showed that the proposed algorithm provides robot car a collision-free path from single- and multi-start point to a target point in an environment with static and moving obstacles. Compared with traditional $\mathrm{A}^{*}$, the proposed algorithm presented a better performance when implemented and tested in realtime environment.
\end{abstract}

Keyword: Algorithm; Autonomous robot car; Dynamic environment; Intelligent path planning; Mobile robot 